\title{
Vacuna anti-varicela
}

\author{
Katia Abarca V.
}

Pontificia Universidad

Católica de Chile

Facultad de Medicina

Departamento de Pediatría

Unidad de Infectología

Recibido: 10-08-05 Aceptado: 17-08-05

Correspondencia a: Katia Abarca Villaseca kabarca@med.puc.cl

\section{Varicella vaccine}

Varicella and herpes zoster represent a significant public health problem. Safe and highly effective varicella vaccines against severe and moderate varicella are currently available. Vaccine efficacy is lower and more variable against mild disease and several risk factors have been associated with mild breakthrough disease. Experts are currently discussing the need for a second vaccine dose. Universal varicella vaccination has been highly effective in reducing morbidity and hospitalizations due to varicella, a strategy that has proven to be cost effective in many regions when the societal-perspective is considered in the analysis. Recent data suggests that varicella vaccination may be associated with an increased incidence of herpes zoster in the elderly. Immunity conferred by varicella vaccination seems to be longlasting but a continued evaluation is needed in order to asses the effect of the changing epidemiology associated with universal immunization.

Key words: varicella; varicella vaccine; efficacy; effectiveness; safety; herpes zoster

Palabras claves: varicela; vacuna anti-varicela; eficacia; efectividad; seguridad; herpes zoster.

\section{Características generales}

$\mathrm{L}$ a vacuna contra la varicela es elaborada con una cepa de virus varicela atenuado, obtenido hace ya varias décadas en Japón por sucesivos pasajes de cultivo de un aislado clínico conocido como cepa OKA. Numerosos estudios previos a su licencia, que incluyeron miles de niños y adultos, demostraron la inmunogenicidad, seguridad y eficacia de esta vacuna. Por ser una vacuna que contiene virus vivo, está contraindicada en sujetos inmunocomprometidos (con la excepción de portadores de VIH en etapa I de la clasificación CDC para la infección por VIH y pacientes con leucemia linfoblástica en etapa de remisión) y en mujeres embarazadas.

Los estudios de inmunogenicidad mostraron excelente seroconversión en niños desde 1 a 12 años con una dosis de vacuna, y con dos dosis en adolescentes y adultos. La vacuna es generalmente bien tolerada, habiéndose reportado fiebre en $14 \%$ de los vacunados (sin diferencias estadísticas con sujetos que recibieron placebo), alteraciones en el sitio de inyección en 15 a $20 \%$ y rash vesiculoso en $4 \%$.

Su seguridad ha sido ratificada después de su licencia y uso universal en países como E.U.A., a través del sistema de vigilancia de eventos adversos asociados a vacuna (Vaccine Adverse Events Reporting SystemVAERS). El evento más reportado es el rash, el cual puede ser debido tanto a la cepa vacunal como a la cepa salvaje. Este rash es generalmente leve y muy poco contagioso; con raras excepciones se han descrito exantemas más extensos con posibilidad de transmisión secundaria observados en niños con leucemia en tratamiento.

\section{Eficacia y fallas de la vacuna}

En estudios pre-licencia fue establecida una eficacia de $85 \%$ contra toda enfermedad y $95-100 \%$ contra enfermedad moderada a grave. La experiencia con su uso masivo post licencia ha mostrado que un mayor porcentaje de vacunados presenta la enfermedad después de 6 semanas de haber sido vacunados (denominados breakthrough cases), por tanto la eficacia contra toda enfermedad es menor a la inicialmente reportada. Estos casos son generalmente leves, con menos de 50 lesiones, predominantemente de tipo máculopapular y dejan pocas cicatrices residuales. Afortunadamente, la protección que otorga la vacuna contra enfermedad moderada a grave continúa siendo elevada.

Una vigilancia activa de 6.314 casos de varicela pudo determinar la contagiosidad de la varicela que ocurre en sujetos vacunados. La contagiosidad fue de la mitad en los casos vacunados comparados con aquellos no vacunados y ella se correlacionó principalmente con la gravedad de la enfermedad: en los 
casos leves la contagiosidad se redujo a un tercio de la observada en los casos graves. Este estudio confirmó la elevada eficacia de la vacuna en prevenir casos moderados y graves (92 a 100\%) ${ }^{1}$.

\section{Vacunación post-exposición y en brotes}

La eficacia de la vacuna administrada post-exposición ha sido establecida en 50 a $67 \%$ si la vacuna se administra en los primeros 3 a 5 días del contacto, con elevada protección contra enfermedad grave $\mathrm{g}^{2,3}$. Un estudio randomizado efectuado en familias, con 22 niños vacunados y 20 que recibieron placebo, demostró similar frecuencia de varicela en ambos grupos, pero la ocurrencia de enfermedad grave fue 8 veces mayor en el grupo placebo ${ }^{4}$.

En un brote en una guardería infantil se utilizó la vacuna observándose varicela en $88 \%$ de los no vacunados comparado con $14 \%$ en los vacunados ${ }^{5}$, con una eficacia de la vacuna similar a la establecida en los estudios pre-licencia.

\section{Impacto de la enfermedad y justificación del uso de vacuna}

Las manifestaciones clínicas de la infección por VVZ (varicela y herpes zoster), se asocian a variadas complicaciones, algunas de las que pueden ser muy graves e incluso llevar a la muerte. Las complicaciones ocurren en 4 a 5,5\% de los casos de varicela, la mayoría de estos sujetos son previamente sanos. Es bien sabido además que la infección en adultos se asocia a mayor morbimortalidad.

Estudios farmacoeconómicos han reforzado la percepción de que esta es una enfermedad relevante y que genera enormes costos, principalmente costos indirectos (pérdidas de días de trabajo). Los estudios de costo efectividad han mostrado que la vacunación no es costo-efectiva desde punto de vista del pagador, pero altamente costo-efectiva desde el punto de vista de la sociedad.

\section{Estrategias de vacunación}

Un país puede decidir el uso universal de la vacuna o sólo vacunar grupos con mayor riesgo, ya sea de desarrollar complicaciones, o de transmitir la enfermedad a personas con alto riesgo de enfermedad grave. Los grupos prioritarios para vacunar son:

- Adolescentes y adultos susceptibles

- Inmunocomprometidos en ciertas condiciones (VIH CDC clase I del CDC, leucemia en remisión)

- Contactos familiares de inmunocomprometidos

- Trabajadores de la salud seronegativos (IgG anti varicela negativa)
- Profesores y empleados de guardería infantiles

- Personal militar susceptible

- Personal e internos de centros institucionalizados

- Mujeres susceptibles en edad fértil, no embarazadas

- Hombres susceptibles que viven con mujeres embarazadas y/o niños

\section{Vacunación universal}

Esta estrategia ha sido implementada en países como E.U.A., Canadá, Japón, Corea, Australia, Finlandia y Uruguay. A continuación se resumen los efectos epidemiológicos observados tras la vacunación universal.

Los cambios observados en la morbi-mortalidad por varicela han sido muy importante en E.U.A., donde la morbilidad se redujo en $76 \%$ el año 1995 y en $87 \%$ en el año 2000. Las hospitalizaciones por varicela se han reducido en $75 \%$, principalmente en los grupos de 0 a 4 años y de 5 a 9 años; asimismo, se ha observado un descenso en los casos de varicela asociados a infecciones graves por Streptococcus pyogenes ${ }^{6-9}$. La mortalidad por varicela en E.U.A. en el período 1990-2001 se redujo en $75 \%$ y ha ocurrido en todos los grupos etarios bajo 50 años, principalmente en los niños de 1 a 4 años $^{10}$.

Diversos autores han mostrado preocupación porque la vacunación universal de niños pudiera tener un efecto poblacional negativo desplazando la enfermedad a edades mayores. Un estudio estimó que el aumento en las coberturas vacunales provocaría un aumento proporcional en los casos de varicela en adultos, pero sin un aumento en la mortalidad global. El aumento en adultos sería menor si se realiza la estrategia de identificar y tratar (catch-up) a niños mayores susceptibles ${ }^{11}$. El CDC confirmó en el año 2003 que con la vacuna universal ha ocurrido un aumento proporcional de los casos de varicela en adultos, pero que ha habido una reducción neta de $27 \%$ en los casos en adultos.

Con el uso universal de la vacuna en E.U.A. se ha ido acumulando evidencias de casos de varicela en vacunados. Se han podido establecer diversos factores asociados a la ocurrencia de enfermedad en vacunados (breakthrough cases). Estos factores son: tiempo entre la vacunación y el contacto (más de 3 a 5 años desde la vacunación implica mayor riesgo), vacunación a edad temprana (antes de 15 meses de edad), vacunación cercana a la vacuna trivírica (menos de 28 días después), uso de corticoesteroides orales en niños asmáticos (vacunación dentro de los 3 meses post uso de corticoesteroides $)^{6-7,12-14}$. Un estudio mostró que títulos de anticuerpos tipo IgG específicos menores a 5 pg ELISA post vacuna se asociaban a 3,5 veces mayor riesgo ${ }^{15}$. Un estudio observó la eficacia de la 
vacuna en el tiempo y según la edad de vacunación, encontrando que la eficacia se reducía de $97 \%$ el primer año de vacunado a $84 \%$ en los siguientes 7 años; la eficacia en el primer año de vacunado dependía de la edad del niño, siendo de $99 \%$ sobre 15 meses de edad y sólo de $73 \%$ en los vacunados antes de los 15 meses de edad ${ }^{16}$. Las fallas de la vacuna en niños que asisten a guarderías parecen depender en gran medida de los niveles de cobertura de la vacuna en estas instituciones. Mientras en 8 guarderías en Israel, en que la cobertura de vacuna no superaba el $40 \%$, la eficacia global de la vacuna fue sólo del 20\% (contra enfermedad moderada a grave la eficacia fue de $93,4 \%)^{17}$, en una escuela en E.U.A. donde más del $95 \%$ de los susceptibles estaba vacunado, la eficacia global de la vacuna fue de $72 \%{ }^{18}$.

Las fallas de la vacuna en niños han puesto en la discusión la necesidad de aplicar una segunda dosis, tema que aún se encuentra en análisis. Un estudio de seguimiento durante 10 años en más de 2.000 niños, la mitad con una dosis y la mitad con dos dosis, mostró que la eficacia en el primer grupo fue de $94,4 \%$ y la del segundo de $98,3 \%$, con 3,3 veces más riesgo de varicela en aquellos vacunados con una dosis ${ }^{19}$.

Otros puntos de preocupación con la introducción de la vacunación universal son la ocurrencia de herpes zoster en vacunados y el posible aumento del herpes zoster en personas mayores no vacunadas. Esto último debido a que se estima que la circulación del virus en una población es el principal estímulo de mantención de la respuesta inmune celular que evita la reactivación viral. Al respecto, varios estudios han demostrado que la tasa de herpes zoster en individuos vacunados es significativamente menor que en los no vacunados. Esto ha sido observado tanto en sujetos sanos, en que las tasas de herpes zoster en no vacunados son de 0,6-1,6/1.000 años persona y en vacunados de 0,18/1.000 años persona, como en inmunocomprometidos: $25 / 1.000$ años persona en no vacunados y $8 / 1.000$ años persona en vacunados ${ }^{20,21}$. Respecto a lo segundo, en E.U.A. entre los años 1998 y 2003 se ha observado efectivamente un aumento de las tasas de herpes zoster de 2,7 a 5,2/10.000 personas, lo que constituye un aumento del $90 \%{ }^{22}$. Por ello se están efectuando estudios de vacunación en adultos para protegerlos del herpes zoster. En un estudio recientemente publicado, randomizado, controlado con placebo, realizado en más de 39.000 adultos sobre 60 años, con una mediana de seguimiento de 3,2 años, se observaron 957 casos de herpes zoster: 315 en vacunados y 642 en el grupo placebo, lo que equivale a una reducción por la vacuna de $61,1 \%(\mathrm{p}<0,001)$. Se observaron 107 casos de neuralgia post-herpética: 27 en vacunados y 80 en el grupo placebo, reducción de $66,5 \%(\mathrm{p}<0,001)$. La vacuna fue bien tolerada y segura en este grupo etario ${ }^{23}$.

\section{Duración de la inmunidad}

En seguimientos de 6, 10 y hasta 20 años se ha observado que persisten tanto los anticuerpos tipo IgG como la inmunidad mediada por células. Sin embargo, es importante evaluar esto a mayor plazo, en especial en condiciones de menor circulación del virus silvestre que puede actuar como refuerzo natural. De acuerdo a lo sucedido con otras vacunas virales como anti-sarampión y anti-rubéola, es posible que se establezca el requerimiento de una dosis de refuerzo.

\section{Resumen}

Varicela y herpes zoster son enfermedades con impacto en salud pública, se dispone actualmente de una vacuna anti-varicela segura y de elevada eficacia contra enfermedad moderada a grave. La protección de la vacuna contra enfermedad leve es menor, variable, y ya se han establecido algunos factores que aumentan el riesgo de fallas. Actualmente está en discusión la necesidad de utilizar una dosis de refuerzo en niños. El uso universal de la vacuna ha demostrado una elevada eficacia en reducir la morbilidad, mortalidad y hospitalizaciones por varicela; la vacunación universal es costo efectiva desde el punto de vista de la sociedad. Se han observado las primeras evidencias de un aumento en la incidencia de herpes zoster en individuos mayores no vacunados. La inmunidad de la vacuna parece ser duradera pero debe seguir en evaluación en contextos epidemiológicos que serán cambiantes por el uso universal de la vacuna.

\section{Referencias}

1.- Seward J F, Zhang J X, Maupin T J, Mascola L, Jumaan A O. Contagiousness of varicella in vaccinated cases: a household contact study. JAMA 2004; 292: 704-8.

2.- Arbeter A M, Starr S E, Plotkin S A.
Varicella vaccine studies in healthy children and adults. Pediatrics 1986; 78: 748-56.

3.- Salzman M B, García C. Postexposure varicella vaccination in siblings of children with active varicella. Pediatr Infect Dis J 1998; 17: 256-7.

4.- Mor M, Harel L, Kahan E, Amir J. Efficacy of postexposure immunization with live attenuated varicella vaccine in the household setting-a pilot study. Vaccine 2004; 23: 325-8.

5.- Izurieta H S, Strebel P M, Blake P A. Postlicensure effectiveness of varicella vaccine during an outbreak in a child care center. JAMA 1997; 278: 1495-9. 
6.- Grose C. Varicella vaccination of children in the United States: assessment after the first decade 1995-2005. J Clin Virol 2005; 33: 89-95.

7.- Vázquez M. Varicella zoster virus infections in children after the introduction of live attenuated varicella vaccine. Curr Opin Pediatr 2004; 16: 80-4.

8.- Patel R A, Binns H J, Shulman S T. Reduction in pediatric hospitalizations for varicella-related invasive group A streptococcal infections in the varicella vaccine era. J Pediatr 2004; 144: 68-74.

9.- Davis M M, Patel M S, Gebremariam A. Decline in varicella-related hospitalizations and expenditures for children and adults after introduction of varicella vaccine in the United States. Pediatrics 2004; 114: 786-92.

10.- Nguyen H Q, Jumaan A O, Seward J F. Decline in mortality due to varicella after implementation of varicella vaccination in the United States. N Engl J Med 2005; 352: 450-8.

11. - Halloran M E, Cochi S L, Lieu T A, Wharton M, Fehrs L. Theoretical epidemiologic and morbidity effects of routine varicella immunization of preschool children in the United States. Am J Epidemiol 1994; 140: 81-104.

12.- Galil K, Lee B, Strine T, Carraher C, Baughman A L, Eaton M, et al. Outbreak of varicella at a day-care center despite vaccination. N Engl J Med 2002; 347: 1909-15.

13.- Galil K, Fair E, Mountcastle N, Britz P, Seward J. Younger age at vaccination may increase risk of varicella vaccine failure. $\mathrm{J}$ Infect Dis 2002; 186: 102-5.

14.- Verstraeten T, Jumaan A O, Mullooly J P, Seward J F, Izurieta H S, DeStefano F, et al A retrospective cohort study of the association of varicella vaccine failure with asthma, steroid use, age at vaccination, and measles-mumps-rubella vaccination. Pediatrics 2003; 112: e98-103.

15.- Li S, Chan I S, Matthews H, Heyse J F, Chan C Y, Kuter B J, et al. Inverse relationship between six week postvaccination varicella antibody response to vaccine and likelihood of long term breakthrough infection. Pediatr Infect Dis J 2002; 21: 337-42.

16. - Vázquez M, LaRussa P S, Gershon A A, Niccolai L M, Muehlenbein C E, Steinberg S P, et al. Effectiveness over time of varicella vaccine. JAMA 2004; 291: 851-5.

17.- Miron D, Lavi I, Kitov R, Hendler A. Vaccine effectiveness and severity of varicella among previously vaccinated children during outbreaks in day-care centers with low vaccination coverage. Pediatr Infect Dis J 2005; 24: 233-6.

18. - Tugwell B, Lee L E, Gillette H, Lorber E M,
Hedberg K, Cieslak P R. Chickenpox outbreak in a highly vaccinated school population. Pediatrics 2004; 113: 455-9.

19. - Kuter B, Matthews H, Shinefield H, Black S, Dennehy P, Watson B, et al. Ten year follow-up of healthy children who received one or two injections of varicella vaccine. Pediatr Infect Dis J 2004; 23: 132-7.

20.- Hardy I, Gershon A A, Steinberg S P, LaRussa P. The incidence of zoster after immunization with live attenuated varicella vaccine. A study in children with leukemia. Varicella Vaccine Collaborative Study Group. N Engl J Med 1991; 325: 1545-50.

21.- Lawrence R, Gershon A A, Holzman R, Steinberg S P. The risk of zoster after varicella vaccination in children with leukemia. N Engl J Med 1988; 318: 543-8.

22.- Yih W K, Brooks D R, Lett S M, Jumaan A O, Zhang Z, Clements K M, et al. The incidence of varicella and herpes zoster in Massachusetts as measured by the Behavioral Risk Factor Surveillance System (BRFSS) during a period of increasing varicella vaccine coverage, 1998-2003. BMC Public Health 2005; 5: 68.

23.- Oxman M N, Levin M J, Johnson G R, Schmader K E, Straus S E, Gelb L D, et al. A vaccine to prevent herpes zoster and postherpetic neuralgia in older adults. $\mathrm{N}$ Engl $\mathrm{J}$ Med 2005; 352: 2271-84 